S. Kwok, M. Dopita, and R. Sutherland, eds.

\title{
Upper Limit to the Deuterium Abundance in Planetary Nebulae
}

\author{
G. Hébrard ${ }^{1}$, D. Péquignot ${ }^{2}$, J.R. Walsh ${ }^{3}$, C. Morisset ${ }^{4}$, A. \\ Vidal-Madjar ${ }^{1}$, R. Ferlet ${ }^{1}$ \\ ${ }^{1}$ Institut d'Astrophysique de Paris, CNRS, 98 bis boulevard Arago, \\ F-75014 Paris, France \\ ${ }^{2}$ Observatoire de Paris-Meudon, F-92195 Meudon Cedex, France \\ ${ }^{3} S T-E C F$, European Southern Observatory, Karl-Schwarzschild Strasse, \\ D-85748 Garching, Germany \\ ${ }^{4}$ Institut d'Astrophysique de Marseille, Traverse du Siphon, BP 8, \\ F-13376 Marseille Cedex 12, France
}

We recently reported the first detection of the deuterium Balmer series in emission in H II regions, performed at the CFHT (Hébrard et al. 2000a) and at the VLT (Hébrard et al. 2000b). These narrow lines are excited by UV continuum fuorescence and arise at the atomic interface between $\mathrm{H}$ iI regions and molecular clouds.

Following this discovery, we used the CFHT with the Gecko spectrograph to observe Balmer lines in planetary nebulae. The non-detection of the deuterium Balmer lines in planetary nebulae represents the first observational evidence that this isotope was, as expected, destroyed in the parent stars.

The data lead to interesting upper limits on the value of $\mathrm{D} / \mathrm{H}$. In the case of NGC 6572 , we obtain a limit of $2.2 \times 10^{-7}$ which is two orders of magnitude below the value usually measured in the interstellar and intergalactic media. This limit compares favorably with even the best limit available $\left(2.5 \times 10^{-7}\right)$ from stellar and solar spectra.

If, on the other hand, the total destruction of deuterium in the parent star of NGC 6572 is taken for granted, then the non-detection of this isotope translates into a $\sim 1 \%$ upper limit to the amount of interstellar gas that could be mixed with the stellar ejecta.

\section{References}

Hébrard, G., Péquignot, D., Vidal-Madjar, A., Walsh, J. R., Ferlet, R., 2000a, A\&A, $354, \mathrm{~L} 79$

Hébrard, G., Péquignot, D., Walsh, J. R., Vidal-Madjar, A., Ferlet, R., 2000b, A\&A, 364, L31 